\title{
Humility in Turkish University Student Athletes and Non-Athletes
}

\author{
Emre Ozan Tingaz ${ }^{1}$ \\ Burcu Güvendi \\ 'Gazi University, Sport Sciences Faculty, Ankara, Turkey and Çă̆ University, Institute of Social Sciences, \\ Department of Psychology, Mersin, Turkey. \\ Email: emreozantingaz@gmail.com Tel: +90507490246 \\ IIstanbul University, Cerrahpaşa Sport Sciences Faculty, Istanbul, Turkey. \\ Email:burcu.guvendi@istanbul.edu.trTel:+905076220381
}

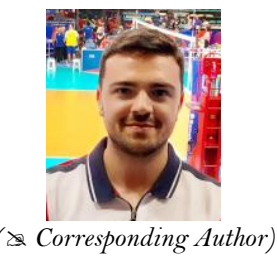

\begin{abstract}
The main purpose of the current research is to examine the humility of university student athletes and non-athletes. In addition, it has been another focus of whether the humility differ according to gender, age, department and monthly expenditure. Furthermore, the humility of the athlete students according to their sports type, sports years and the best athletic scores was investigated. In the present study, two different public universities from Istanbul and Ankara, aged between 18, 23 (Mage $=20.49$ years SD $=1.35), 54.3 \%(\mathrm{n}=222)$ were female and $45.7 \%(\mathrm{n}=187)$ were male, a total of four hundred nine athlete and non-athlete students were included through convenience sampling. The instrument selected for the study was the Humility Scale which has been developed by Elliott (2010) and adapted to Turkish by Sarıçam et al. (2012). The total score and subdimensions of humility in the present study did not differ significantly by gender, age, monthly expenditure, athletic status (athletes or non-athletes), sport type (team or individual sports) and sport year. On the other hand, in the sub-dimension of focus on others, it was found that the students in the primary teaching department had significantly higher scores than the physical education and sports teacher students. In modest self-assessment sub-scale, the average score of athletes who have the best athletic score in a Turkey competition higher than athletes who have the best athletic score in a regional competition.
\end{abstract}

Keywords: Humility, Humble, University student, Student athlete, University student athlete, University department.

Citation | Emre Ozan Tingaz; Burcu Güvendi (2019). Humility in Turkish University Student Athletes and Non-Athletes. Asian Journal of Education and Training, 5(3): 459-466.

History:

Received: 19 June 2019

Revised: 26 July 2019

Accepted: 2 September 2019

Published: 8 October 2019

Licensed: This work is licensed under a Creative Commons Attribution 3.0 License (oc))

Publisher: Asian Online Journal Publishing Group

\begin{abstract}
Acknowledgement: Both authors contributed to the conception and design of the study.

Funding: This study received no specific financial support.

Competing Interests: The authors declare that they have no conflict of interests.

Transparency: The authors confirm that the manuscript is an honest, accurate, and transparent account of the study was reported; that no vital features of the study have been omitted; and that any discrepancies from the study as planned have been explained.

Ethical: This study follows all ethical practices during writing.
\end{abstract}

\section{Contents}

1. Introduction

2. Method.

3. Findings

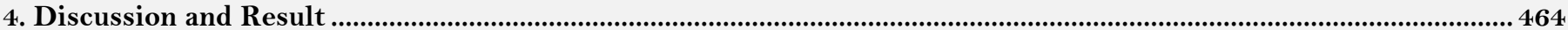

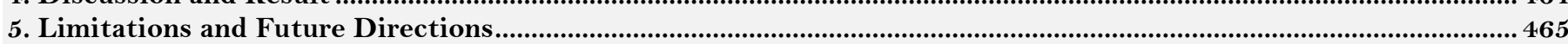

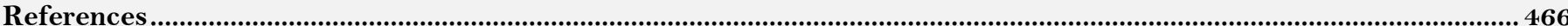




\section{Contribution of this paper to the literature}

This study contributes to the existing literature by examining the humility of university student athletes and non-athletes.

\section{Introduction}

Humility is not about being arrogant, self-centered or jealous, but about being respectful and open-minded (Peters et al., 2011). In other words, humility is a desirable personal trait that reflects the desire to understand self, expressed as identity, strengths, and boundaries, and is linked to the perspective of the self in relation to others (Nielsen et al., 2010). Hökelekli (2007) remarks that humility is something very different in the case of failure or negative personality stuck into a sense of self. It means not to consider itself superior in a deep perspective and to have a moderate self-perception. He additionally explain that the authority, wealth, talent, fame, bodily strength and beauty, success, such as to come to give more importance to temporary things, to see them as a means of service and help to the development and maturation of himself and others. Seligman (2002) points out that humility is an important pattern in controlling self-control and negative desires of the self, preventing self-justification according to the wishes of the self, and contributing to the ability to conduct beneficial behaviors towards others. A humble personality, thanks to his/her deep inner world, loves and cares for other people, sees them as superior as he/she deserves and values them very much. According to Topçu (2012) the humble individual who acts regardless of earthly values belonging to others, avoids affectation and unpretentious behaviors, acts according to reality, and does not get false pride and grandiosity about himself. The greatest motivation that drives him to engage in such words and behaviors is his mature personality. Humble individuals see their mistakes as a part of human life and are therefore very open to the offer of assistance directed at correcting their mistakes (Elliott, 2010).

When the field literature is examined, it is seen that the researches on humility are limited in the field of sports (McNamee, 2002; Brymer and Oades, 2009; Austin, 2014; Foster, 2019). On the other hand, when examined in other studies conducted with sports, Sevde and Tuncel (2012) concluded that tolerance is an indispensable necessity in preventing negativities such as disrespect, insight, fighting and violence in sports. Çağlayan et al. (2017) advocate the view that the level of forgiveness of the students doing sports was higher than those who did not. Türkçapar and Karademir (2016) examined the modesty levels of individuals who took formation education and reported that the moderation level of the formation group students who participated in the study was above average. While there was no difference in humility among students according to income level and age variables, it was determined that women's scores were significantly higher than men's. Aksoy et al. (2017) in the study in which the university students examined the levels of happiness and humility; It was determined that gender, age, number of siblings, place of birth, place of residence during the university education, class level and parental education level did not make a significant difference in the humility level of the students, and that the place where the majority of life was spent caused a significant difference. They further point out that there was no significant relationship between students' happiness and humility levels. Gediksiz (2013) concluded that there is no significant relationship between humility and psychological well-being. He additionally noted that humility levels of university students did not show significant differences in terms of gender, perceived income level, parental attitudes.

\section{Method}

\subsection{Participants}

In the present study, two different public universities from Istanbul and Ankara, aged between 18-23 (Mage = 20.49 years $\mathrm{SD}=1.35), 54.3 \%(\mathrm{n}=222)$ were female and $45.7 \%(\mathrm{n}=187)$ were male, a total of four hundred nine athlete and non-athlete students were included through convenience sampling.

\subsection{Instruments}

The instrument selected for the study was the Humility Scale which has been developed by Elliott (2010) and adapted to Turkish by Sarıçam et al. (2012). The scale has 13 items and four factors called as openness, selfforgetfulness, modest self-assessment and focus on others. The items were rated on a 5 -point scale $(1=$ completely disagree, 5 = completely agree). 1, 2, 3, 4, 5, 6. Items are reverse scored. The lowest score that can be obtained from the scale is 13 and the highest score is 60. The high score obtained from the scale is related to high humility. Internal consistency coefficient stated as .63 for openness sub-dimension, .67 for self-forgetfulness sub-dimension, .72 for modest self-assessment sub-dimension, .79 for focus on others sub dimension and .81 for whole scale.

\subsection{Statistics}

The statistical analyses were carried out using SPSS 23. Initially it was determined whether there were missing and mistaken data. Total scores of the participants' humility scale were calculated and outlier analysis was performed. Then, skewness and kurtosis coefficients were calculated for the purpose of normality analysis. The kurtosis and skewness coefficients were found to be within \pm 1 range for both scale total score and subscales. Parametric tests were preferred because histogram and $Q-Q$ Plot graphs did not deviate from normal distribution.

Independent sample $t$ test was carried out to determine whether the total and subscale scores of the humility scale showed a significant difference according to gender, whether or not to do sports, and sports types. One Way ANOVA was carried out to determine whether the total and subscale scores of the humility scale showed a significant difference according to the participants' department, monthly expenditure, sports year and athletic scores. Bonferroni test from post-hoc tests was carried out for significant differences. Probabilities below $p=0.05$ were regarded as significant. 


\section{Findings}

Table-1. Demographic characteristics of participants.

\begin{tabular}{|c|c|c|c|}
\hline Variable & & $\mathbf{n}$ & $\%$ \\
\hline \multirow[t]{6}{*}{ Age } & 18,00 & 27 & 7,5 \\
\hline & 19,00 & 62 & 17,2 \\
\hline & 20,00 & 89 & 24,7 \\
\hline & 21,00 & 97 & 26,9 \\
\hline & 22,00 & 59 & 16,3 \\
\hline & 23,00 & 27 & 7,5 \\
\hline \multirow{6}{*}{ Department } & Physical education and sport teaching & 87 & 22.3 \\
\hline & Trainer education & 37 & 9.5 \\
\hline & Sport management & 25 & 6.4 \\
\hline & Recreation & 56 & 14.3 \\
\hline & Special education teaching & 23 & 5.9 \\
\hline & Primary school teaching & 163 & 41.7 \\
\hline \multirow[t]{4}{*}{ Monthly expenditure } & $0-500$ TL $(0-75 €)$ & 149 & 36.4 \\
\hline & $501-1000(75-150 €)$ & 156 & 38.1 \\
\hline & $1001-1500(150-225 €)$ & 76 & 18.6 \\
\hline & $1501+(225+€)$ & 28 & 6.8 \\
\hline \multirow[t]{2}{*}{ Athletic status } & Athletes & 181 & 44.3 \\
\hline & Non-athletes & 228 & 55.7 \\
\hline \multirow[t]{2}{*}{ Sport type } & Team sports & 99 & 57.9 \\
\hline & Individual sports & 72 & 42.1 \\
\hline \multirow[t]{5}{*}{ Sports year } & 0-1 year & 19 & 10.5 \\
\hline & 2-4 years & 56 & 30.9 \\
\hline & $5-7$ years & 48 & 26.5 \\
\hline & $8-10$ years & 31 & 17.1 \\
\hline & $10+$ years & 27 & 14.9 \\
\hline \multirow[t]{4}{*}{ The best athletic scores } & No & 53 & 32.7 \\
\hline & Province competition & 30 & 18.5 \\
\hline & Region competition & 34 & 21.0 \\
\hline & Turkey competition & 45 & 27.8 \\
\hline
\end{tabular}

As shown in Table 1; 21.3\% $(\mathrm{n}=87)$ of Physical Education and Sports Teaching, $9 \%(\mathrm{n}=37)$ of Trainer Education, $6.1 \%(\mathrm{n}=25)$ of Sports Management, $13.7 \%(\mathrm{n}=56)$ of Recreation, $5.6 \%(\mathrm{n}=23)$ of them are in Special Education Teaching and $39.9 \%(\mathrm{n}=163)$ are in Primary School Teaching Department. When the monthly expenditures are examined; 36.4\% $(\mathrm{n}=149)$ 0-500 TL $(0-75 €), 38.1 \%(\mathrm{n}=156) 501-1000 \mathrm{TL}(75-150 €), 18.6 \%(\mathrm{n}$ $=76)$ of $1001-1500 \mathrm{TL}(75-225 €), 6.8 \%(\mathrm{n}=28)$ of which $1500+\mathrm{TL}(225+€)$ is spent. The amount of expenditure is categorized as national scholarship $(500 \mathrm{TL}-75 €)$. While $44.3 \%(\mathrm{n}=181)$ of the students were athletes, $55.7 \%(\mathrm{n}$ $=228)$ were not athletes. While $57.9 \%(\mathrm{n}=99)$ of the athletes do football, basketball, volleyball and handball team sports, $42.1 \%(\mathrm{n}=72)$ do individual sports such as athletics, judo, swimming, taekwondo, karate and wrestling. The distribution of the number of people in the branches was categorized as individual and team sports due to the imbalance. When the sports years are examined; $10.5 \%(\mathrm{n}=19)$ 0-1 years, 30.9\% $(\mathrm{n}=56) 2-4$ years, $26.5 \%(\mathrm{n}=48)$ 5-7 years, $17.1 \%(\mathrm{n}=31)$ 8-10 years. $14.9 \%(\mathrm{n}=27)$ stated that they had been doing sports for more than 10 years. When the best top three athletic scores are examined, 32.7\% $(\mathrm{n}=53)$ does not have a place in top three, $18.5 \%$ $(\mathrm{n}=30)$ has top three in a province competition, $21 \%(\mathrm{n}=34)$ has top three in a region competition and $27.8 \%(\mathrm{n}=45)$ has top three in a Turkey competition.

Table-2. T-test result of total and sub-scales of humility by gender.

\begin{tabular}{c|c|c|c|c|c|c}
\hline \multicolumn{2}{c}{ Table-2. T-test result of total and sub-scales of humility by gender. } \\
\hline \multirow{2}{*}{ Hotal } & Gender & $\mathbf{N}$ & $\mathbf{M}$ & $\mathbf{S D}$ & $\mathbf{t}$ & $\mathbf{p}$ \\
\hline \multirow{2}{*}{ Openness } & Female & 222 & 41.08 & 3.98 & .170 & .865 \\
\cline { 2 - 7 } & Male & 187 & 41.01 & 3.70 & & \\
\hline \multirow{2}{*}{ Self-forgetfulness } & Female & 222 & 9.21 & 1.93 & .179 & .858 \\
\cline { 2 - 8 } & Male & 187 & 9.17 & 2.04 & & \\
\hline \multirow{2}{*}{ Modest self-assessment } & Female & 222 & 11.27 & 2.39 & 1.149 & .248 \\
\cline { 2 - 8 } & Male & 187 & 11.01 & 2.19 & & \\
\hline & Female & 222 & 9.30 & 2.33 & 1.471 & .142 \\
\hline \multirow{2}{*}{ Focus on others } & Male & 187 & 9.65 & 2.41 & & \\
\hline & Female & 222 & 11.28 & 2.26 & .499 & .618 \\
\hline & Male & 187 & 11.17 & 2.28 & & \\
\hline
\end{tabular}

When Table 2 was examined, it can be said that the total score of humility $(t=.170, p>.05)$ and subscales of openness $(t=.179, \mathrm{p}>.05)$, self-forgetfulness $(\mathrm{t}=1.149, \mathrm{p}>.05)$, modest self-assessment $(\mathrm{t}=1.471, \mathrm{p}>.05)$ and focus on others $(\mathrm{t}=.499, \mathrm{p}>.05)$ did not differ significantly by gender. 
Table-3. One way ANOVA results of total and sub-scales of humility by age.

\begin{tabular}{|c|c|c|c|c|c|c|}
\hline \multicolumn{2}{|l|}{ Humility } & $\mathbf{N}$ & $\mathbf{M}$ & SD & $\mathbf{F}$ & p \\
\hline \multirow{7}{*}{ Total } & 18.00 & 27 & 42.25 & 3.49 & \multirow{7}{*}{2.132} & \multirow{7}{*}{.061} \\
\hline & 19.00 & 62 & 41.64 & 4.19 & & \\
\hline & 20.00 & 89 & 40.62 & 3.83 & & \\
\hline & 21.00 & 97 & 40.55 & 3.74 & & \\
\hline & 22.00 & 59 & 41.23 & 3.96 & & \\
\hline & 23.00 & 27 & 42.51 & 3.42 & & \\
\hline & Total & 361 & 41.14 & 3.87 & & \\
\hline \multirow{7}{*}{ Openness } & 18.00 & 27 & 9.22 & 1.88 & \multirow{7}{*}{.460} & \multirow{7}{*}{.806} \\
\hline & 19.00 & 62 & 9.46 & 1.98 & & \\
\hline & 20.00 & 89 & 9.06 & 1.94 & & \\
\hline & 21.00 & 97 & 9.14 & 1.76 & & \\
\hline & 22.00 & 59 & 9.35 & 2.14 & & \\
\hline & 23.00 & 27 & 9.44 & 2.04 & & \\
\hline & Total & 361 & 9.24 & 1.93 & & \\
\hline \multirow{7}{*}{ Self-forgetfulness } & 18.00 & 27 & 11.48 & 2.31 & \multirow{7}{*}{1.391} & \multirow{7}{*}{.227} \\
\hline & 19.00 & 62 & 11.09 & 2.38 & & \\
\hline & 20.00 & 89 & 10.79 & 2.22 & & \\
\hline & 21.00 & 97 & 11.09 & 2.26 & & \\
\hline & 22.00 & 59 & 11.22 & 2.62 & & \\
\hline & 23.00 & 27 & 12.07 & 2.12 & & \\
\hline & Total & 361 & 11.14 & 2.33 & & \\
\hline \multirow{7}{*}{ Modest self-assessment } & 18.00 & 27 & 9.77 & 2.45 & \multirow{7}{*}{.580} & \multirow{7}{*}{.715} \\
\hline & 19.00 & 62 & 9.69 & 2.44 & & \\
\hline & 20.00 & 89 & 9.37 & 2.46 & & \\
\hline & 21.00 & 97 & 9.24 & 2.15 & & \\
\hline & 22.00 & 59 & 9.67 & 2.38 & & \\
\hline & 23.00 & 27 & 9.18 & 2.23 & & \\
\hline & Total & 361 & 9.45 & 2.34 & & \\
\hline \multirow{7}{*}{ Focus on others } & 18.00 & 27 & 11.77 & 2.02 & \multirow{7}{*}{.983} & \multirow{7}{*}{.428} \\
\hline & 19.00 & 62 & 11.38 & 2.26 & & \\
\hline & 20.00 & 89 & 11.39 & 2.37 & & \\
\hline & 21.00 & 97 & 11.07 & 2.33 & & \\
\hline & 22.00 & 59 & 10.98 & 2.24 & & \\
\hline & 23.00 & 27 & 11.81 & 2.09 & & \\
\hline & Total & 361 & 11.29 & 2.27 & & \\
\hline
\end{tabular}

As can be seen in Table 3, it was found that the total score and the subscales of the humility did not differ significantly by age ( $\mathrm{p}>.05)$.

Table-4. One way ANOVA results of total and sub-scales of humility by department.

\begin{tabular}{|c|c|c|c|c|c|c|c|}
\hline & Humility & $\mathbf{N}$ & $\mathbf{M}$ & SD & $\mathbf{F}$ & $p$ & Bonferroni \\
\hline \multirow{6}{*}{ Total } & PE and sport teaching & 87 & 40.80 & 3.53 & \multirow{6}{*}{1.131} & \multirow{6}{*}{.344} & \multirow{6}{*}{-} \\
\hline & Trainer education & 37 & 41.32 & 3.88 & & & \\
\hline & Sport management & 25 & 42.08 & 5.01 & & & \\
\hline & Recreation & 56 & 40.35 & 3.73 & & & \\
\hline & Special education teaching & 23 & 42.04 & 4.26 & & & \\
\hline & Primary school teaching & 163 & 41.00 & 3.80 & & & \\
\hline \multirow{6}{*}{ Openness } & PE and sport teaching & 87 & 9.40 & 2.11 & \multirow{6}{*}{.711} & \multirow{6}{*}{.615} & \multirow{6}{*}{-} \\
\hline & Trainer education & 37 & 9.40 & 1.99 & & & \\
\hline & Sport management & 25 & 9.04 & 2.09 & & & \\
\hline & Recreation & 56 & 8.83 & 2.27 & & & \\
\hline & Special education teaching & 23 & 9.26 & 2.24 & & & \\
\hline & Primary school teaching & 163 & 9.09 & 1.79 & & & \\
\hline \multirow{6}{*}{ Self-forgetfulness } & PE and sport teaching & 87 & 11.21 & 2.15 & \multirow{6}{*}{.923} & \multirow{6}{*}{.466} & \multirow{6}{*}{-} \\
\hline & Trainer education & 37 & 11.54 & 2.11 & & & \\
\hline & Sport management & 25 & 10.56 & 2.45 & & & \\
\hline & Recreation & 56 & 11.10 & 2.48 & & & \\
\hline & Special education teaching & 23 & 11.65 & 2.62 & & & \\
\hline & Primary school teaching & 163 & 10.98 & 2.32 & & & \\
\hline \multirow{6}{*}{$\begin{array}{l}\text { Modest self- } \\
\text { assessment }\end{array}$} & PE and sport teaching & 87 & 9.58 & 2.35 & \multirow{6}{*}{1.679} & \multirow{6}{*}{.139} & \multirow{6}{*}{-} \\
\hline & Trainer education & 37 & 8.81 & 1.89 & & & \\
\hline & Sport management & 25 & 10.52 & 2.32 & & & \\
\hline & Recreation & 56 & 9.35 & 2.60 & & & \\
\hline & Special education teaching & 23 & 9.69 & 2.09 & & & \\
\hline & Primary school teaching & 163 & 9.42 & 2.42 & & & \\
\hline \multirow{6}{*}{ Focus on others } & PE and sport teaching (a) & 87 & 10.59 & 2.34 & \multirow{6}{*}{2.632} & \multirow{6}{*}{$.023^{*}$} & \multirow{6}{*}{$f>a$} \\
\hline & Trainer education (b) & 37 & 11.56 & 1.96 & & & \\
\hline & Sport management (c) & 25 & 11.96 & 2.18 & & & \\
\hline & Recreation $(d)$ & 56 & 11.05 & 2.46 & & & \\
\hline & Special education teaching (e) & 23 & 11.43 & 2.65 & & & \\
\hline & Primary school teaching $(\mathrm{f})$ & 163 & 11.50 & 2.12 & & & \\
\hline
\end{tabular}

$\overline{\mathrm{PE}}=$ Physical education; ${ }^{*} p<.05$. 
When Table 4 is examined, it is observed that the $\mathrm{F}$ value is not significant of the total score of humility $\left(\mathrm{F}_{(5-}\right.$ $\left.{ }_{385)}=.1 .131 ; \mathrm{p}>.05\right)$, openness $\left(\mathrm{F}_{(5-385)}=.711 ; \mathrm{p}>.05\right)$, self-forgetfulness $\left(\mathrm{F}_{(5-385)}=.923 ; \mathrm{p}>.05\right)$, modest self-assessment $\left(\mathrm{F}_{(5-385)}=.1 .679 ; \mathrm{p}>\right.$.05). However the $\mathrm{F}$ value is significant for the subscale of focus on others $\left(\mathrm{F}_{(5-385)}=.2 .632 ; \mathrm{p}<.05\right)$. The homogeneity of the variances was tested to determine which groups belong to the difference $(p=.400, p>.05)$. Bonferroni test was carried out because of does not require equal sample number, exhibit the differences between groups consistency and avoided I and II type errors (Miller, 1969).

It can be seen that the difference between the mean scores of the sub-dimension of the primary school students' focus on others $(\mathrm{M}=11.50, \mathrm{SD}=2.12)$ and the mean score of the students of physical education and sports teachers $(\mathrm{M}=10.59, \mathrm{SD}=2.34)$ were significant $(\mathrm{p}<.05)$. However, there is no significant difference between trainer education $(\mathrm{M}=11.56, \mathrm{SD}=1.96)$, sport management $(\mathrm{M}=11.96, \mathrm{SD}=2.18)$, recreation $(\mathrm{M}=11.05, \mathrm{SD}=2.46)$ and special education teaching $(\mathrm{M}=11.43, \mathrm{SD}=2.65)$.

Table-5. One way ANOVA results of total and sub-scales of humility by monthly expenditure

\begin{tabular}{|c|c|c|c|c|c|c|}
\hline \multicolumn{2}{|c|}{ Humility } & $\mathbf{N}$ & $\mathbf{M}$ & SD & $\mathbf{F}$ & p \\
\hline \multirow[t]{4}{*}{ Total } & $0-500 \mathrm{TL}(0-75 €)$ & 149 & 41.30 & 3.78 & \multirow{4}{*}{.547} & \multirow{4}{*}{.651} \\
\hline & $501-1000(75-150 €)$ & 156 & 41.03 & 3.78 & & \\
\hline & $1001-1500(150-225 €)$ & 76 & 40.81 & 4.22 & & \\
\hline & $1501+(225+€)$ & 28 & 40.42 & 3.62 & & \\
\hline \multirow[t]{4}{*}{ Openness } & $0-500$ TL $(0-75 €)$ & 149 & 9.06 & 1.81 & \multirow{4}{*}{.985} & \multirow{4}{*}{.400} \\
\hline & $501-1000(75-150 €)$ & 156 & 9.41 & 2.05 & & \\
\hline & $1001-1500(150-225 €)$ & 76 & 9.05 & 2.26 & & \\
\hline & $1501+(225+€)$ & 28 & 9.07 & 1.58 & & \\
\hline \multirow[t]{4}{*}{ Self-forgetfulness } & $0-500$ TL $(0-75 €)$ & 149 & 11.50 & 2.34 & \multirow{4}{*}{2.467} & \multirow{4}{*}{.062} \\
\hline & $501-1000(75-150 €)$ & 156 & 10.79 & 2.25 & & \\
\hline & $1001-1500(150-225 €)$ & 76 & 11.25 & 2.37 & & \\
\hline & $1501+(225+€)$ & 28 & 11.10 & 2.04 & & \\
\hline \multirow[t]{4}{*}{ Modest self-assessment } & $0-500$ TL $(0-75 €)$ & 149 & 9.57 & 2.43 & \multirow{4}{*}{1.772} & \multirow{4}{*}{.152} \\
\hline & $501-1000(75-150 €)$ & 156 & 9.64 & 2.33 & & \\
\hline & $1001-1500(150-225 €)$ & 76 & 9.18 & 2.45 & & \\
\hline & $1501+(225+€)$ & 28 & 8.67 & 1.90 & & \\
\hline \multirow[t]{4}{*}{ Focus on others } & $0-500$ TL $(0-75 €)$ & 149 & 11.16 & 2.14 & \multirow{4}{*}{.317} & \multirow{4}{*}{.813} \\
\hline & $501-1000(75-150 €)$ & 156 & 11.19 & 2.34 & & \\
\hline & $1001-1500(150-225 €)$ & 76 & 11.32 & 2.21 & & \\
\hline & $1501+(225+€)$ & 28 & 11.57 & 2.74 & & \\
\hline
\end{tabular}

As can be seen in Table 5, total score and subscales of the humility scale did not change according to monthly expenditure $(\mathrm{p}>.05)$.

Table-6. T-test result of total and sub-scales of humility by athletic status.

\begin{tabular}{|c|c|c|c|c|c|c|}
\hline Humility & Athletic status & $\mathbf{N}$ & $\mathbf{M}$ & SD & $\mathrm{t}$ & p \\
\hline \multirow[t]{2}{*}{ Total } & Athlete & 181 & 41.19 & 3.69 & .663 & .507 \\
\hline & Non-athlete & 228 & 40.93 & 3.98 & & \\
\hline \multirow[t]{2}{*}{ Openness } & Athlete & 181 & 9.29 & 2.04 & .883 & .378 \\
\hline & Non-athlete & 228 & 9.11 & 1.93 & & \\
\hline \multirow[t]{2}{*}{ Self-forgetfulness } & Athlete & 181 & 11.39 & 2.19 & 1.826 & .069 \\
\hline & Non-athlete & 228 & 10.97 & 2.38 & & \\
\hline \multirow[t]{2}{*}{ Modest self-assessment } & Athlete & 181 & 9.25 & 2.41 & 1.558 & .120 \\
\hline & Non-athlete & 228 & 9.62 & 2.33 & & \\
\hline \multirow[t]{2}{*}{ Focus on others } & Athlete & 181 & 11.24 & 2.33 & .129 & .897 \\
\hline & Non-athlete & 228 & 11.21 & 2.23 & & \\
\hline
\end{tabular}

When Table 6 examined it can be seen that there is no significant difference total score of humility scale $(\mathrm{t}=$ $.663, \mathrm{p}>.05)$, openness $(\mathrm{t}=.883, \mathrm{p}>.05)$, self-forgetfulness $(\mathrm{t}=1.826, \mathrm{p}>.05)$, modest self-assessment $\mathrm{t}=1.558, \mathrm{p}>$ $.05)$ and focus on others subscales $(\mathrm{t}=.129, \mathrm{p}>.05)$.

Table-7. T-test result of total and sub-scales of humility by athletic status by type of sports.

\begin{tabular}{c|c|c|c|c|c|c}
\hline Humility & Type of sports & $\mathbf{N}$ & $\mathbf{M}$ & $\mathbf{S D}$ & $\mathbf{t}$ & $\mathbf{p}$ \\
\hline \multirow{2}{*}{ Total } & Team sports & 99 & 41.39 & 3.99 & 1.136 & .258 \\
\cline { 2 - 7 } & Individual sports & 72 & 40.76 & 3.25 & & \\
\hline \multirow{2}{*}{ Openness } & Team sports & 99 & 9.41 & 2.10 & .861 & .391 \\
\cline { 2 - 7 } & Individual sports & 72 & 9.13 & 2.00 & & \\
\hline \multirow{2}{*}{ Self-forgetfulness } & Team sports & 99 & 11.34 & 2.22 & .134 & .894 \\
\cline { 2 - 7 } & Individual sports & 72 & 11.38 & 2.14 & & \\
\hline \multirow{2}{*}{ Fodest self-assessment } & Team sports & 99 & 9.25 & 2.34 & .192 & .848 \\
\cline { 2 - 7 } & Individual sports & 72 & 9.18 & 2.50 & & \\
\hline
\end{tabular}

When Table 7 examined it can be seen that there is no significant difference total score of humility scale $(t=$ $1.136, \mathrm{p}>.05)$, openness $(\mathrm{t}=.861, \mathrm{p}>.05)$, self-forgetfulness $(\mathrm{t}=.134, \mathrm{p}>.05)$, modest self-assessment $(\mathrm{t}=.192, \mathrm{p}>$ $.05)$ and focus on others subscales $(t=.903, \mathrm{p}>.05)$. 
Table-8. One way ANOVA results of total and sub-scales of humility by sports year.

\begin{tabular}{|c|c|c|c|c|c|c|}
\hline \multicolumn{2}{|l|}{ Humility } & $\mathbf{N}$ & $\mathbf{M}$ & SD & $\mathbf{F}$ & p \\
\hline \multirow[t]{5}{*}{ Total } & O-1 year & 19 & 40.10 & 3.79 & \multirow{5}{*}{1.149} & \multirow{5}{*}{.335} \\
\hline & $2-4$ years & 56 & 41.55 & 3.66 & & \\
\hline & $5-7$ years & 48 & 40.66 & 3.53 & & \\
\hline & $8-10$ years & 31 & 41.16 & 3.52 & & \\
\hline & $10+$ years & 27 & 42.03 & 4.04 & & \\
\hline \multirow[t]{5}{*}{ Openness } & 0-1 year & 19 & 9.31 & 2.21 & \multirow{5}{*}{1.101} & \multirow{5}{*}{.358} \\
\hline & $2-4$ years & 56 & 9.66 & 1.90 & & \\
\hline & $5-7$ years & 48 & 8.97 & 1.90 & & \\
\hline & $8-10$ years & 31 & 8.90 & 2.24 & & \\
\hline & $10+$ years & 27 & 9.51 & 2.20 & & \\
\hline \multirow[t]{5}{*}{ Self-forgetfulness } & 0-1 year & 19 & 11.00 & 2.51 & \multirow{5}{*}{1.805} & \multirow{5}{*}{.130} \\
\hline & 2-4 years & 56 & 11.51 & 2.09 & & \\
\hline & 5-7 years & 48 & 10.93 & 1.89 & & \\
\hline & $8-10$ years & 31 & 11.38 & 2.45 & & \\
\hline & $10+$ years & 27 & 12.25 & 2.17 & & \\
\hline \multirow[t]{5}{*}{ Modest self-assessment } & 0-1 year & 19 & 9.15 & 2.21 & \multirow{5}{*}{1.662} & \multirow{5}{*}{.161} \\
\hline & $2-4$ years & 56 & 8.82 & 2.36 & & \\
\hline & 5-7 years & 48 & 9.35 & 2.46 & & \\
\hline & $8-10$ years & 31 & 10.12 & 2.77 & & \\
\hline & $10+$ years & 27 & 8.92 & 1.81 & & \\
\hline \multirow[t]{5}{*}{ Focus on others } & O-1 year & 19 & 10.63 & 2.19 & \multirow{5}{*}{.996} & \multirow{5}{*}{.411} \\
\hline & $2-4$ years & 56 & 11.55 & 2.41 & & \\
\hline & 5-7 years & 48 & 11.39 & 2.33 & & \\
\hline & $8-10$ years & 31 & 10.74 & 2.40 & & \\
\hline & $10+$ years & 27 & 11.33 & 2.13 & & \\
\hline
\end{tabular}

As can be seen in Table 8, it was found that the total score and the subscales of the humility did not differ significantly according to the sport year $(\mathrm{p}>.05)$.

Table-9. One way ANOVA results of total and sub-scales of humility by the best athletic scores.

\begin{tabular}{|c|c|c|c|c|c|c|c|}
\hline \multicolumn{2}{|c|}{ Humility } & $\mathbf{N}$ & $\mathbf{M}$ & SD & $\mathbf{F}$ & p & Bonferroni \\
\hline \multirow[t]{4}{*}{ Total } & No & 53 & 41.56 & 3.44 & \multirow{4}{*}{.348} & \multirow{4}{*}{.791} & \multirow{4}{*}{ - } \\
\hline & Province competition & 30 & 40.86 & 4.03 & & & \\
\hline & Region competition & 34 & 41.35 & 3.79 & & & \\
\hline & Turkey competition & 45 & 40.93 & 3.73 & & & \\
\hline \multirow[t]{4}{*}{ Openness } & No & 53 & 9.60 & 1.81 & \multirow{4}{*}{1.218} & \multirow{4}{*}{.305} & \multirow{4}{*}{ - } \\
\hline & Province competition & 30 & 8.80 & 2.09 & & & \\
\hline & Region competition & 34 & 9.55 & 1.94 & & & \\
\hline & Turkey competition & 45 & 9.15 & 2.38 & & & \\
\hline \multirow[t]{4}{*}{ Self-forgetfulness } & No & 53 & 11.71 & 1.98 & \multirow{4}{*}{.868} & \multirow{4}{*}{.459} & \multirow{4}{*}{ - } \\
\hline & Province competition & 30 & 11.33 & 2.32 & & & \\
\hline & Region competition & 34 & 11.55 & 2.32 & & & \\
\hline & Turkey competition & 45 & 11.02 & 2.28 & & & \\
\hline \multirow[t]{4}{*}{ Modest self-assessment } & No $(a)$ & 53 & 8.96 & 2.10 & \multirow{4}{*}{3.542} & \multirow{4}{*}{$.016^{*}$} & \multirow{4}{*}{$d>c$} \\
\hline & Province competition (b) & 30 & 9.90 & 2.68 & & & \\
\hline & Region competition (c) & 34 & 8.38 & 2.11 & & & \\
\hline & Turkey competition (d) & 45 & 9.84 & 2.54 & & & \\
\hline \multirow[t]{4}{*}{ Focus on others } & No & 53 & 11.28 & 2.33 & \multirow{4}{*}{1.433} & \multirow{4}{*}{.235} & \multirow{4}{*}{ - } \\
\hline & Province competition & 30 & 10.83 & 2.35 & & & \\
\hline & Region competition & 34 & 11.85 & 2.46 & & & \\
\hline & Turkey competition & 45 & 10.91 & 2.06 & & & \\
\hline
\end{tabular}

When Table 9 examined, it can be seen that there is a not significant $\mathrm{F}$ value total score of humility $\left(\mathrm{F}_{(3-}\right.$ $\left.{ }_{158)}=.348 ; \mathrm{p}>.05\right)$, openness $\left(\mathrm{F}_{(3-158)}=1.218 ; \mathrm{p}>.05\right)$, self-forgetfulness $\left(\mathrm{F}_{(3-158)}=.868 ; \mathrm{p}>.05\right)$ and focus on others $\left(\mathrm{F}_{(3-}\right.$ $\left.{ }_{158)}=1.433 ; \mathrm{p}>.05\right)$. However there is significant $\mathrm{F}$ value self-assessment subscale $\left(\mathrm{F}_{(3-158)}=3.542 ; \mathrm{p}<.05\right)$. The homogeneity of the variances was tested to determine which groups belong to the difference $(\mathrm{p}=.379, \mathrm{p}>.05)$ and Bonferroni test was carried out.

Mean scores of self-assessment subscale of athletes who has the best score in a Turkey competition $(\mathrm{M}=9.84$, $\mathrm{SD}=2.54)$ significantly higher than has the best score in a regional competition $(\mathrm{M}=8.38, \mathrm{SD}=2.11)$. There is no significant diffrence between the athletes who does not have any top three score mean $(\mathrm{M}=8.96, \mathrm{SD}=2.10)$ and athletes who has the best score in a province competition $(\mathrm{M}=9.90, \mathrm{SD}=2.68)$.

\section{Discussion and Result}

The main purpose of the current research is to examine the humility of university student athletes and nonathletes. In addition, it has been another focus of whether the humility differ according to gender, age, department and monthly expenditure. Furthermore, the humility of the athlete students according to their sports type, sports years and the best athletic scores was investigated. While interpreting the findings, both studies on humility and studies on narcissism were evaluated.

In the light of the findings, the total score and sub-dimensions of humility in the present study did not differ significantly by gender. Similarly, in another study with university students, it was reported that the total score of humility did not differ according to gender (Aksoy et al., 2017). Guven (2019) also stated in his doctoral 
dissertation with 2557 adult sample group that the total score of humility did not differ significantly according to gender. Exline and Geyer (2004) also found that there was no significant relationship between gender and humility. Similarly, Vural (2016) and Gediksiz (2013) stated that total score of humility did not change according to gender in their master thesis. Elliott (2010) the original owner of the scale used in this study, also reported that there was no significant difference between gender and humility. However, in another study, a significant difference was found in the self-assessment sub-dimension of university students in favor of male and in favor of female in the sub-dimensions of focus on others, but it was reported that general humility did not differ significantly according to gender (Karademir, 2019). Türkçapar and Karademir (2016) indicated that the sub-dimensions did not differ significantly by gender, but that the total score of humility was significantly higher than that of females. The majority of the studies examined were not interested in the sub-dimensions that were processed with the total score of humility. In this respect, it can be considered that there is no significant difference in the total score of humility in terms of gender and it is similar to the findings in our present study.

Another independent variable of the study was age that there was no significant difference between the total score of humility and its subscales. Guven (2019) in his doctoral dissertation with 18-65 participants, found that the total score of humility by age did not change significantly. However, many studies have reported that humility does not differ significantly by age (Elliott, 2010; Türkçapar and Karademir, 2016; Vural, 2016; Aksoy et al., 2017).

According to the departments included in the study, it was examined whether the humility differed or not. In the sub-dimension of focus on others, it was found that the students in the primary teaching department had significantly higher scores than the physical education and sports teacher students. It was also found that the mean score of total humility did not differ significantly in the other sub-dimensions. When the items of the subdimension of focus on others are examined, it can be thought that the individual expresses humility through others. It was found that the focus on others sub-dimension had a positive correlation with empathy (Elliott, 2010). Accordingly, Ekinci and Aybek (2010) compared the empathic tendencies of university students in different departments and found that the empathic tendencies of primary school students were higher than those of philosophy teachers. However, it can also be considered that empathy skills may be more developed since classroom teacher students are interested in the younger age group. They may be considered to have a higher score than the sub-dimension of focusing on others, as they may be more empathic.

It was found that the total score of humility and the total score obtained from the sub-dimensions did not differ significantly according to the monthly expenditure situation. Vural (2016) stated that humility does not differ significantly according to income status, which may be due to the high number of middle income participants in the study. Gediksiz (2013) also found that the total score of humility did not change according to the income level in his master's thesis. Türkçapar and Karademir (2016) also found that neither humility total score nor its subdimensions differed significantly according to family income level. The findings are similar to the current results.

The total scores of the students' humility and sub-dimensions did not differ significantly according to whether they are athletes or non-athletes. However, considering that there is a negative relationship between humility and narcissism (Guven, 2019) it is among the findings that total narcissism scores of athletes are higher than nonathletes (Tazegül and Güven, 2015). Demirel et al. (2018) found that exhibitionism, which is the sub-dimension of narcissism, was significantly higher in athletes than non-athletes. However, there was no significant difference in other sub-dimensions. Vaughan et al. (2019) were also stated that expert athletes scored higher than non-athletes on narcissism in their research.

The humility total scores of the students who are athletes and the scores of the sub-dimensions do not differ significantly according to the sports types. Cavusoglu et al. (2017) also found that the level of narcissism did not differ significantly according to the sports branch. However, Vaughan et al. (2019) were stated that individual athletes scored higher than team athletes on narcissism. It can be the different culture may be the reason.

The humility total scores of the students who are athletes and the scores of the sub-dimensions do not differ significantly according to the sports year. Whether the sport year is 1 year or more than 10 years, humility does not differ significantly. The reason for this is that, based on the findings of scores, it can be thought that the scores in this process are more effective in humility than in the sports year.

The humility total scores of the students who are athletes, the openness, self-forgetfulness and focus on the others do not differ significantly according to athletic scores. But in modest self-assessment sub-scale, the average score of athletes who have the best athletic score in a Turkey competition higher than athletes who have the best athletic score in a regional competition. In fact, this finding seems to be proof of a Turkish proverb, "Empty spike stands upright". In this metaphor, the spike is likened to a human being, and it is expressed that it can stand upright when it is empty and its head will bend forward when it is full. Considering that humility can also be highly influenced by cultural characteristics, the humility of athletes with a higher athletic score may also be higher. On the other hand, the superiority complex versus the inferiority complex that Adler (2004) mentions in the theory of individual psychology may be another reason. According to Adler, more or less inferiority lies beneath the superiority complex. Accordingly, athletes with a lower athletic score may be considered less humble due to the superiority complex.

In summary, the total score and sub-dimensions of humility in the present study did not differ significantly by gender, age, monthly expenditure, athletic status (athletes or non-athletes), sport type and sport year. However, in the sub-dimension of focus on others, it was found that the students in the primary teaching department had significantly higher scores than the physical education and sports teacher students. In modest self-assessment subscale, the average score of athletes who have the best athletic score in a Turkey competition higher than athletes who have the best athletic score in a regional competition.

\section{Limitations and Future Directions}

The current study is characterized by a number of limitations. The study relied on the Turkish sample and the study group relatively small. Related to this issue, it can be considered different culture and large study groups for the future studies. Different variables that can predict to humility for university student athletes or elite athletes can be take into account for the future studies. 


\section{References}

Adler, A., 2004. The meaning and purpose of life. Istanbul: Say.

Aksoy, A.B., A. Güngör Aytar and N. Kaytez, 2017. Investigation of happiness and humility levels of university students according to some variables. Kastamonu Education Magazine, 25(3): 1119-1132.

Austin, M.W., 2014. Is humility a virtue in the context of sport? Journal of Applied Philosophy, 31(2): 203-214.Available at: https://doi.org/10.1111/japp.12049.

Brymer, E. and L.G. Oades, 2009. Extreme sports: A positive transformation in courage and humility. Journal of Humanistic Psychology, 49(1): 114-126.Available at: https://doi.org/10.1177/0022167808326199.

Çağlayan, H.S., M. Akandere and Ö. Gül, 2017. The effect of physical activities on the level of forgiveness of university students. International Journal of Educational Sciences, 11: 314-322.

Cavusoglu, G., A.K. Yılmaz, M. Kabadayı, S.H. Abacı and M.Y. Tasmektepligıl, 2017. Comparison of narcissism levels of students in the faculty of sports sciences in terms of some demographic variables. Turkish Journal of Sport and Exercise, 19(2): 150-156.Available at: https://doi.org/10.15314/tsed.296702.

Demirel, H., M. Altın, Y.G. Yalçın and H. Demir, 2018. Comparison of narcissism levels of university students who do sports and do not do sports. Turkish Journal of Sport Sciences, 1(2): 72-77.

Ekinci, Ö. and B. Aybek, 2010. Examination of prospective teachers' empathic and critical thinking dispositions. Elementary School Online, 9(2): 3-14.Available at: https://doi.org/10.17522/nefefmed.57817.

Elliott, J.C., 2010. Humility: Development and analysis of a scale (Doctoral Dissertation). Available from https://trace.tennessee.edu/cgi/viewcontent.cgi? article=1887\&context=utk_graddiss.

Exline, J.J. and A.L. Geyer, 2004. Perceptions of humility: A preliminary study. Self and Identity, 3(2): 95-114.Available at: https://doi.org/10.1080/13576500342000077.

Foster, C., 2019. Sport science: Progress, hubris, and humility. International Journal of Sports Physiology and Performance, 14(2): 141143.Available at: https://doi.org/10.1123/ijspp.2018-0982.

Gediksiz, E., 2013. Examining the relationship between modesty and psychological well-being in terms of various variables. Unpublished master's Thesis. Sakarya University, Institute of Educational Sciences, Sakarya.

Guven, M., 2019. The relationship between narcissism, humility and religiosity (Doctoral Dissertation, Atatürk University, Social Sciences Institute).

Hökelekli, H., 2007. A value: Humility. DEM Journal, 1(2): 114-119.

Karademir, N., 2019. Investigating the humility levels of the geography department students. Kahramanmaraş Sütçü İmam University Journal of Social Sciences, 16(1): 181-200.Available at: https://doi.org/10.33437/ksusbd.511315.

McNamee, M., 2002. Hubris, humility, and humiliation: Vice and virtue in sporting communities. Journal of the Philosophy of Sport, 29(1): 38-53.Available at: https://doi.org/10.1080/00948705.2002.9714621.

Miller, R.G., 1969. Simultaneous statistical inference. New York: McGraw-Hill.

Nielsen, R., J.A. Marrone and H.S. Slay, 2010. A new look at humility: Exploring the humility concept and its role in socialized charismatic leadership. Journal of Leadership \& Organizational Studies, 17(1): 33-43.Available at: https://doi.org/10.1177/1548051809350892.

Peters, A.S., W.C. Rowat and M.K. Johnson, 2011 . Associations between dispositional humility and social relationship quality. Psychology, 2(3): 155-161.Available at: https://doi.org/10.4236/psych.2011.23025.

Sarıçam, H., A. Akın, E. Gediksiz and U. Akın, 2012. The Turkish form of the modesty scale: Validity and reliability study. Kalem Journal of Education and Human Sciences, 2(2): 165-188

Seligman, M.E.P., 2002. Authentic happiness. New York, NY: Free.

Sevde, M. and S.D. Tuncel, 2012. Athletes' perceptions of the concept of hos tolerance in sport per [perception of athletes concern in tolarance in sport 'term]. SPORMETRE Journal of Physical Education and Sports Sciences, 10(4): 125-129.Available at: https://doi.org/10.1501/sporm_0000000229.

Tazegül, Ü. and Ö. Güven, 2015. Comparison of the levels of narcissism of bodybuilding athletes and elite athletes and non-athletes in different sports branches. International Journal of Social Science, 33: 465-473.Available at: https://doi.org/10.9761/jasss2770.

Topçu, N., 2012. Morals. 3rd Edn., Istanbul: Dervish.

Türkçapar, Ü. and T. Karademir, 2016. Investigation of the humility levels of individuals undergoing training in terms of different variables [training in terms of some variables]. Journal of Sport and Performance Research, 7(1): 21-28.Available at: https://doi.org/10.17155/spd.75424.

Vaughan, R., D.J. Madigan, G.L. Carter and A.R. Nicholls, 2019. The dark triad in male and female athletes and non-athletes: Group differences and psychometric properties of the short dark triad (SD3). Psychology of Sport and Exercise, 43: 64-72.Available at: https://doi.org/10.1016/j.psychsport.2019.01.002.

Vural, M.E., 2016. The relationship between humility, piety and psychological well-being in adults. Unpublished Master's Thesis. Marmara University, Institute of Social Sciences, Istanbul. 